\title{
THE IMPACT OF KEY TEMPERATURE MEASURING POINTS ON THERMAL ERROR COMPENSATION MODEL TRANSFER BETWEEN MILLING CENTERS OF THE SAME PRODUCT LINE
}

\author{
M. Straka ${ }^{1}$, M. Mares $^{1 *}$, O. Horejs ${ }^{1}$ \\ ${ }^{1}$ Czech Technical University in Prague, Faculty of Mechanical Engineering, Department of Production Machines and \\ Equipment, RCMT, Horska 3, 12800 Prague, Czech Republic \\ *Corresponding author; e-mail:m.mares@rcmt.cvut.cz
}

\begin{abstract}
Machine tool thermal errors are an important element in machined workpiece inaccuracies. In the past few decades, thermal errors have been successfully reduced by software compensation techniques such as multiple linear regression analysis, finite element method, neural network and transfer function (TF). A phenomenological approach based on TFs is used for thermal error compensation in this research. This approach respects basic heat transfer mechanisms in the MT structure and requires a minimum of additional gauges. Every MT is unique due to manufacturing inaccuracies in components, assembly processes (different preloads in bolts, deviations in the assembly and seating of components, etc.) or working environment (air-conditioned room or ordinary production hall). The aim of this paper is to investigate thermal error compensation model transfer between machines of the same product line to improve thermal error model prediction. Several milling centers of the same product line were tested with varying non-stationary activities of heat sources from linear axis movements and in varying working environments. The issue of key temperature point selection is considered.
\end{abstract}

\section{Keywords:}

Thermal error; Compensation; Machine tool; Key temperature points; Transferability

\section{INTRODUCTION}

The heat generated e.g. by moving axes and machining processes creates thermal gradients, resulting in the thermal elongation and bending of machine tool (MT) elements, which substantially deteriorate MT accuracy. Consequently, up to $75 \%$ of all geometrical errors of machined workpieces are caused by temperature effects [Mayr 2012]. Thermal errors may be sufficiently reduced by new MT design concepts which are less sensitive to thermal effects. This type of intervention in the MT structure leads to a pareto set of different parameters and designers have to concentrate on preserving other MT properties as well [Grossmann 2015]. At the same time, redesigning MT structure is usually possible in the prototype phase of new products. Adaptive or intelligent control of cooling systems [Hellmich 2018], integrated additional sensors in the MT structure [Naumann 2018] or direct (in-process) measurement techniques [Zimmermann 2020] may also be very efficient in minimising thermo-mechanical impacts on MT accuracy, but they do increase machine and operationa costs and result in machining process interruptions and prolonged production time. A very promising contemporary approach is the use of FEM coupled with model order reduction (MOR) techniques to reduce computing time [Hernandez-Becerro 2018]. The problem of boundary condition complexity at the machine or component level, however, is still present in this solution. In contrast, indirect (software) compensation of thermal errors at the tool centre point (TCP) is one of the most widely employed reduction techniques due to its cost-effectiveness and ease of application. Different types of software error compensation are becoming a crucial part of contemporary technological development in the context of Industry 4.0 and machine tool intelligentisation [Liu 2019].

Ordinarily, approximation models are based on measured auxiliary variables [Brecher 2004] (temperature, spindle speed, etc.) used to calculate the resulting thermally induced displacements at the TCP. Many strategies have been investigated to establish these models, e.g. multiple linear regressions (MLR) [Srinivas 2017], artificial neural networks (ANN) [Mize 2000], transfer functions (TF) [Brecher 2004], etc. The majority of the compensation models introduced in the literature have the potential to significantly reduce MT thermal errors. The methods differ in the amount and type of input variables, and training and modelling time required for composition and model structure architecture (white, black or grey-boxes [Li 2008]). Therefore, further efforts should be focused on the applicability and verification of approaches in real industrial conditions and environments. 
Tab. 1: Description of target machine differences.

\begin{tabular}{|c|c|c|c|c|c|}
\hline $\begin{array}{l}\text { Machine } \\
\text { tool no. }\end{array}$ & $\begin{array}{l}\text { Preload of } Y \text { and } Z \\
\text { axis ball-screws }\end{array}$ & $\begin{array}{l}\text { Lubricant } \\
\text { for axes }\end{array}$ & Cooling of feed drives & Placement & $\begin{array}{l}\text { Date of } \\
\text { experiments }\end{array}$ \\
\hline 1 & $2.1 \mathrm{kN}$ & & $\begin{array}{l}12 \mathrm{~kW} \text { (spindle and } A \text { axis } \\
\text { - compressor cooler) }\end{array}$ & Laboratory & 2021/03 \\
\hline 2 & & NLGI 00 & $8 \mathrm{~kW}(A$ axis - compressor & Show room & 2019/06 \\
\hline 3 & $1.7 \mathrm{kN}$ & & cooler), $0.56 \mathrm{~kW} / \mathrm{K}$ (spindle & Production hall & $2019 / 07$ \\
\hline 4 & & ISO VG 100 & - surge cooler) & Show room & $2015 / 07$ \\
\hline
\end{tabular}

Impact of different spindle units on thermal behaviour and modelling effort within machines of the same production line was presented in [Horejš 2020]. Thermal errors caused by the activity of linear motion axes $Y$ and $Z$ of four 5-axis CNC milling centres are considered in the modelling effort in this research. These target machines are from the same production line but differ in the adjustment of components (lubrication, cooling systems, and pre-loading), location (laboratory, show-room, and production hall) and date of the experiments. The main aim of the article is to analyse the conditions of compensation model transferability between the target machines. The impact of three key temperature measuring points (motor of motion axis, motor flange, and ball screw nut bearings) on compensation model effectivity is taken into account.

First, the target machines and their differences are described in more detail. Next, three compensation models (based on different measured key temperature points) are calibrated using measured data from one target machine. An analysis of the transferability of the compensation models to the other machines is given in the next section. Finally, the results are critically discussed from the practical, experimental and modeling effort points of view.

\section{TARGET MACHINES}

The four target machines for this article are gantry type 5axis milling centres of the same production line. A schema of the target machines with workspace dimensions is depicted in Fig. 1.

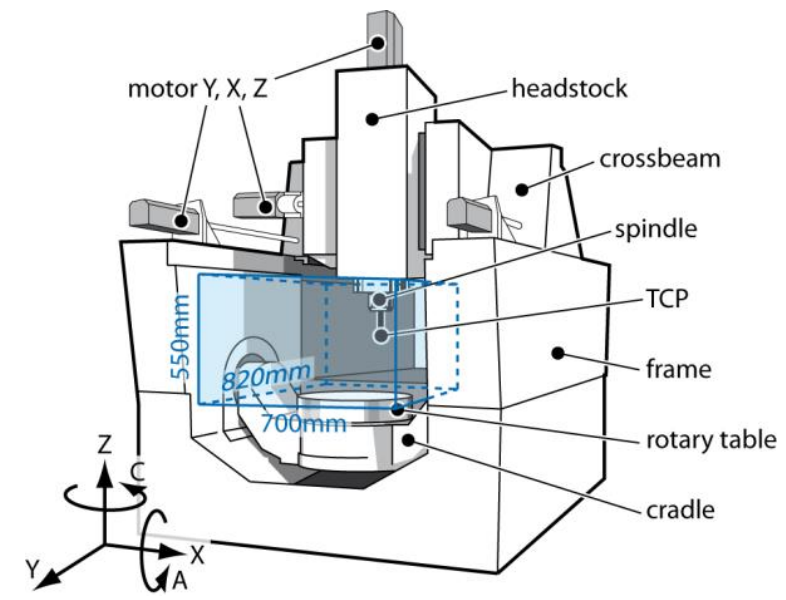

Fig. 1: Schema of the target machines - gantry type 5-axis milling centres - with workspace dimensions.

Three of the four machines were brand new in the experimental period; the measurements were performed in a laboratory environment or a MT producer's showroom. One machine is located in a production hall and has been used there for some years. The experiments differ by the date on which they were implemented on the target machines. Other differences pertain to pre-loading of ball screws, lubrication and cooling systems. The differences between the target machines are summarised in Table 1. The results of the experiments also enable classification of the thermo-mechanical behavior of similar machines in terms of component wear, assembly and manufacturing inaccuracy. MT no. 1 is considered for calibration of the compensation models, and the rest of the MTs for analysis of the transferability of the models.

\section{CALIBRATION, IDENTIFICATION AND MODELLING}

The research aims to construct compensation models of thermal errors between the table and TCP caused by movement in linear axes and examine the effectivity after their transfer to other machines of the same production line. Temperature inputs from different key measuring points into the compensation model are considered:

- A compensation model using motor temperatures as input parameters was introduced in [Mareš 2020]. These internal temperatures are embedded in the MT control system, could have an indirect relationship to MT thermal errors (measurement of electromotor winding) and a resolution of $1^{\circ} \mathrm{C}$ (they are used primarily for safety, diagnostic and maintenance purposes).

- A compensation model using ball screw bearings as input parameters was introduced in [Mareš 2019]. Target machines are not standardly equipped with these sensors; the sensors are placed on the moving parts of the machine and have very good correlation with thermal errors caused by movement in MT linear axes (the placement is close to the heat source).

- A flange of a linear axis motor is the third key measuring point considered in the research. The flange is close to both thermal sources (motor and ball screw) and is firmly connected to the MT structure. The flange is a stationary part of the MT. The solution requires an external temperature sensor.

A schema of the kinematic chain of motion axes $Y$ and $Z$ with the approximate position of temperature inputs into the compensation model is depicted in the following figure.

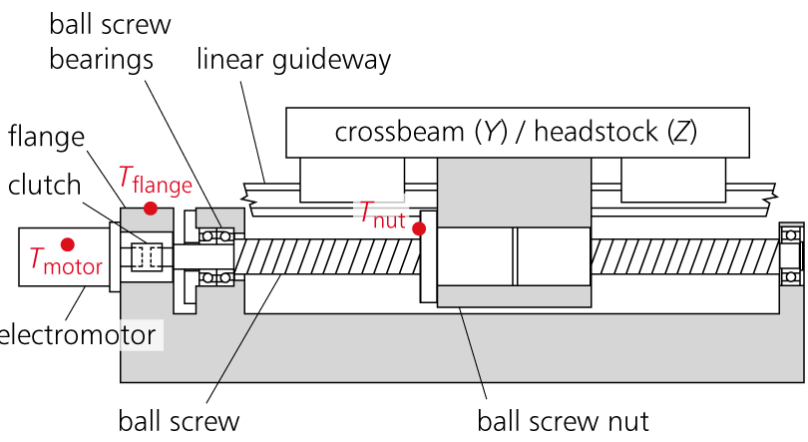

Fig. 2: Schema of kinematic chain of motion axes of target machines with approximate temperature probe positions. 


\subsection{Modelling approach based on transfer functions}

The concept behind the modelling approach lies in usage of a minimum of additional gauges (the only information from the MT control system [Brecher 2004]), an open structure that is easy to extend and modify (advantageous for machine learning principles and intelligent solutions within the MT [Blaser 2019]), real time application and ease of implementation into MT control systems. A set of TFs appears to be a suitable tool.

A compensation strategy based on TFs is a dynamic method with a physical basis. A discrete TF is used to describe the link between the excitation and its response:

$y(t)=u(t) \cdot \varepsilon+e(t)$,

$y(t)=\frac{a_{n} Z^{-n}+\cdots+a_{1} Z^{-1}+a_{0} Z^{0}}{b_{m} Z^{-m}+\cdots+b_{1} Z^{-1}+b_{0} Z^{0}} u(t) ;$ where $m>n$.

The vector $u(t)$ in equations (1) and (2) is the TF input in the time domain, $y(t)$ is the output vector in the time domain, $\varepsilon$ represents the TF in the time domain, $e(t)$ is the disturbance value (further neglected), $a_{n}$ is the calibration coefficient of the TF input, $b_{m}$ is the calibration coefficient of the TF output, $n$ is the order of the TF numerator, $m$ is the order of the TF denominator, and $z$ is a complex number. The differential form of the TF (generally suitable for programming languages) is introduced in equation (3),

$$
\begin{aligned}
y(k)= & \frac{u(k-n) a_{n}+\cdots+u(k-1) a_{1}+u(k) a_{0}}{b_{0}}- \\
& -\frac{y(k-m) b_{m}+\cdots+y(k-1) b_{1}}{b_{0}}
\end{aligned}
$$

where $k-n(k-m)$ signifies the $n$-multiple (m-multiple) delay in sampling frequency. Linear parametric models of autoregressive with external input (ARX) or output error $(\mathrm{OE})$ identifying structures are used with the help of Matlab Identification Toolbox [Ljung 2020]. The ARX as an optimal model structure (with the best fitting quality and robustness) is discussed in [Mayr 2018].

Excitations in the case of the employed TFs mean temperatures measured close to heat sinks or sources and the responses stand for the linear deflections in the examined directions. The approximation quality of the simulated behaviour is expressed by a local peak-to-peak approach ( $p 2 p$, differences of maximum and minimum simulated or measured deformations) and a global approach based on the least square method (equation (4)).

fit $=\left(1-\frac{\left\|Z_{\text {mea. }}-Z_{\text {sim. }}\right\|}{\left\|Z_{\text {mea. }}-\bar{Z}_{\text {sim. }}\right\|}\right) \cdot 100$

The $Z_{\text {mea }}$ value in equation (4) is the measured output (thermal displacements in the Z-direction), $Z_{\text {sim. }}$ is the simulated/predicted model output, and $\bar{Z}_{\text {mea }}$ expresses the arithmetic mean of the measured output over time. The fit is a percentage value where $100 \%$ would equal a perfect match of measured and simulated behaviours.

\subsection{Calibration test set-ups and conditions}

Data was acquired during the experiments using a NI cRIO9014 produced by National Instruments and LabVIEW software. The NI cRIO-9014 collected NC data from the machine tool control systems via the profibus communication standard.

Data processing and the identification process, as well as MT thermal behaviour modelling, were performed in Matlab and Matlab Simulink (version R2017a). All of the results and conclusions are associated with the following experiment conditions. The models were valid for no-load conditions (e.g. cutting process). The models describing axis speed thermo-mechanical impacts were calibrated in one MT axial configuration (at the centre of the table), and compensation was realised in linear directions only. This article is focused on deformations in the $Z$ direction caused by movement on the $Y(Z T Y)$ and $Z(Z T Z)$ axes. Other directions are beyond the scope of this article and subject to similar conclusions.

Generally, calibration should describe a transient characteristic between two thermodynamic equilibria; one with the MT surroundings and one with all active thermal sinks and sources that are to be approximated.

Eddy current sensors firmly clutched in the measuring fixture were employed for noncontact sensing of the displacements between the TCP of a test mandrel (length $125 \mathrm{~mm}$, diameter $40 \mathrm{~mm}$ ) and the rotary table (regular position of the workpiece), per ISO 230-3 international standard [ISO 230-3 2007], as shown in Chyba! Nenalezen zdroj odkazů.. The displacements were sensed in micrometre resolution.

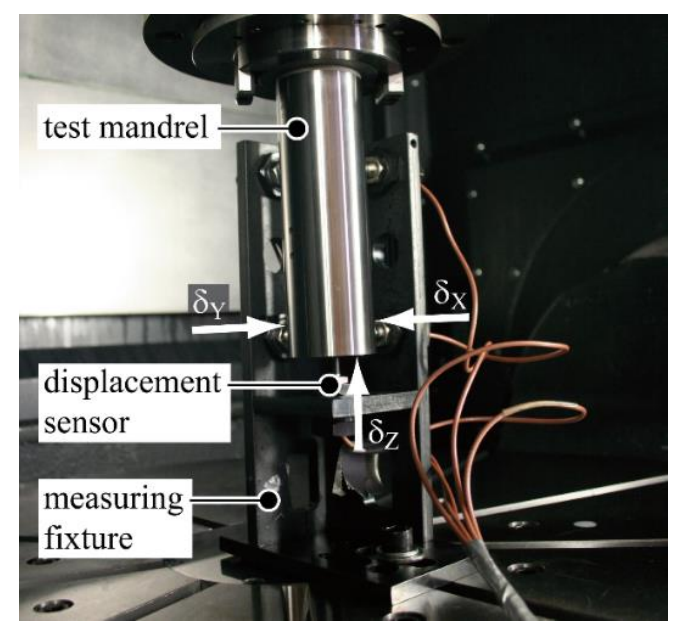

Fig. 3: Experimental setup in the MT workspace.

Measurement of movement in linear axes is divided into two cyclically repeated sections: measurement of test mandrel thermal displacements (10 $\mathrm{s}$ in the measuring fixture; position 1 in Fig. 4) and a 20-minute repeated load application. In the second section, the MT conducts a cyclical linear ( $Y$ or $Z$ axis) motion in trajectories that are under a specific constant speed (positions 3 and 4 in Fig. 4). The experiment set-up for $Y$-axis movement impact inspection (with all positions, trajectories and speeds) is depicted in Fig. 4.

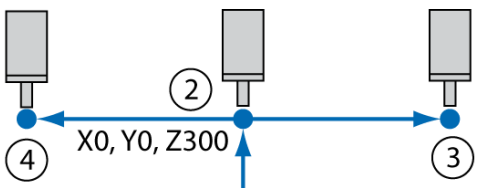

$X 0, Y-300, Z 300$

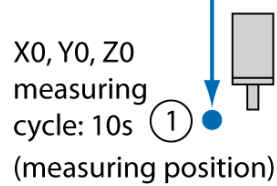

$\mathrm{X} 0, Y 300, Z 300$

$$
\begin{aligned}
& \text { loading cycle: } \\
& \text { repetition } \\
& \text { (3) 4 } 4 \\
& 20 \text { min }
\end{aligned}
$$

Fig. 4: Experiment set-up for calibration of $Y$-axis movement impact on MT thermal behaviour.

The input temperature behaviours measured during the calibration test on MT no. 1 are depicted in Fig. 5. The axis speed was set to a constant $10000 \mathrm{~mm} \cdot \mathrm{min}^{-1}$. Only the heating phase is measured; linear dependency of the inputs 
and outputs during $Y$ and $Z$ axis movements are expected and will be discussed in the following chapters.

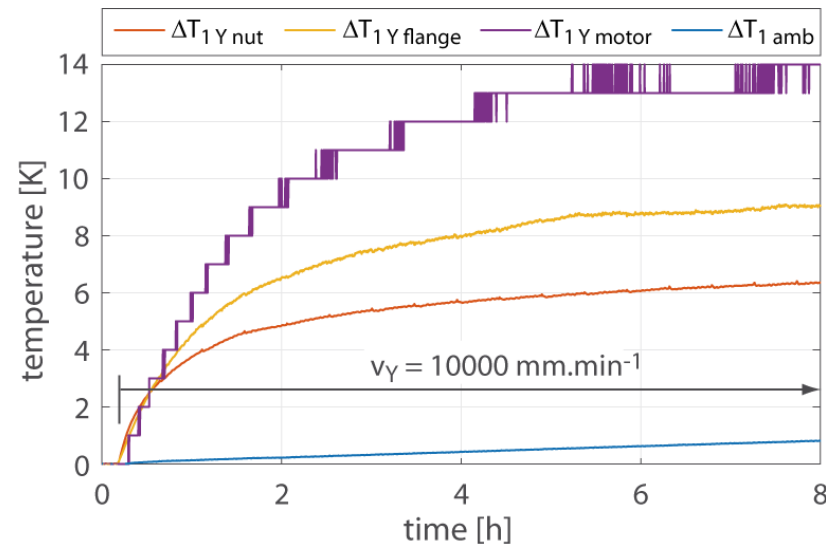

Fig. 5: Calibration test set-up for $Y$-axis movement impact on MT no. 1, conditions and input temperature behaviours.

\subsection{Identification of models approximating thermal errors caused by linear axis movement}

The measured deformation ZTY during the calibration test on MT no. 1, along with the TF identification process, is shown in Fig. 6. The graph shows the following outputs: measured (mea. ZTY) and simulated (identified TFs with temperature inputs of nut, flange and motor key positions) along with the fit value.

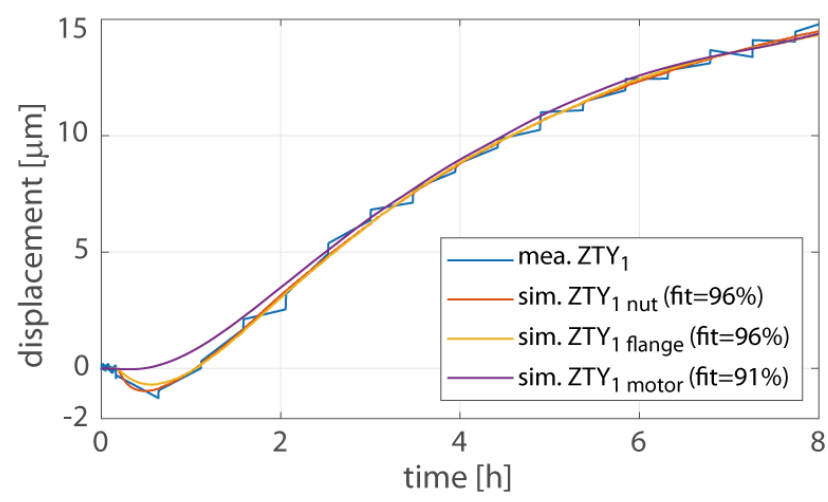

Fig. 6: Measured and simulated outputs from the thermomechanical system during the calibration test.

The TF models of thermal deformations ZTY of MT no. 1 depending on three measured key temperature points (nut, flange and motor) are expressed by equations (5), (6) and (7) respectively.

sim.ZTY 1 nut $=\Delta T_{1 Y \text { nut }} \cdot \varepsilon_{Y \text { nut }}$,

sim. $Z T Y_{1 \text { flange }}=\Delta T_{1 Y \text { flange }} \cdot \varepsilon_{Y \text { flange }}$,

sim. $Z T Y_{1 \text { motor }}=\Delta T_{1 Y \text { motor }} \cdot \varepsilon_{Y \text { motor }}$.

Values sim. $Z T Y_{1 \text { nut, }} \operatorname{sim} . Z T Y_{1 \text { flange }}$ and $\operatorname{sim} . Z T Y_{1 \text { motor in }}$ equations (5), (6) and (7) respectively are the simulated outputs from the thermo-mechanical system of MT no.1 (displacements), $\Delta T_{1} \mathrm{Y}$ nut, $\Delta T_{1} \mathrm{Y}$ flange and $\Delta T_{1} \mathrm{Y}$ motor are the input key temperatures expressed in relative coordinates, and $\varepsilon \curlyvee$ nut,$\varepsilon Y$ flange and $\varepsilon Y$ motor are the identified TFs in the time domain. The stability of the identified TFs and a better understanding of $Y$-axis movement impact on MT no.1's thermo-mechanical behaviour are expressed by the LTI step response depicted in the Fig. 7. System excitation represents the sudden change of the key temperature equal to $1 \mathrm{~K}$ (the black curve on the graph), and the system responses are the predicted deformations given by equation (5), (6) and (7) (red, yellow and purple curves on the graph). The established calibration coefficients $a_{n}$ and $b_{\mathrm{m}}$ of the identified TFs are summarised in Table 2. The order of the TF was selected based on the best fit value.

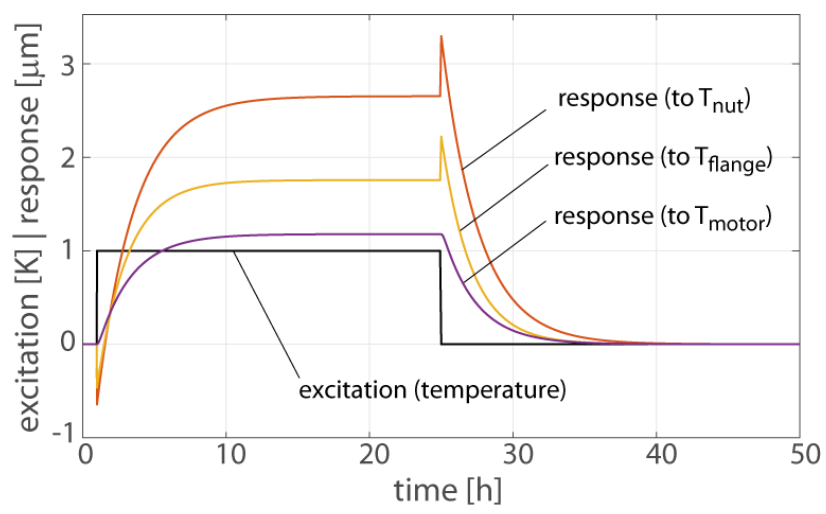

Fig. 7: Step responses of the identified TFs.

The impact of ambient temperature is neglected in the models since the experiments were quite short and the surroundings were stable during the experiment implementation periods.

Tab. 2: Coefficients of the identified TF describing Y-axis movement impact on thermal error in the $Z$ direction.

\begin{tabular}{|c|c|c|c|}
\hline TF & & Coefficients & \\
\hline & $a_{0}$ & $a_{1}$ & $a_{2}$ \\
\hline$\varepsilon Y$ nut & -0.643184758 & 0.6434694129 & 0 \\
\hline$\varepsilon Y$ flange & -0.464350315 & 0.4645814159 & 0 \\
\hline \multirow[t]{2}{*}{$\varepsilon Y$ motor } & $2.97448314 \mathrm{e}-07$ & 0 & 0 \\
\hline & $\boldsymbol{b}_{0}$ & $\boldsymbol{b}_{1}$ & $\boldsymbol{b}_{2}$ \\
\hline EY nut & 1 & -0.999892737 & 0 \\
\hline$\varepsilon Y$ flange & 1 & -0.99986841 & 0 \\
\hline$\varepsilon \gamma$ motor & 1 & -1.997757465 & 0.997757718 \\
\hline
\end{tabular}

\section{EVALUATION OF MODEL EFFECTIVITY}

Four experiments were carried out to examine the limits of transferability of the compensation models based on three measured key temperatures. Modified compensation models were applied to all of the target machines.

\subsection{Experimental set-ups}

A list of the four $Y$-axis movement verification experiments is presented in Table 3 . The first verification test was realised on calibrated MT no. 1 (with a different axis speed from the calibration test) to examine the linear dependency between the inputs and outputs of the thermo-mechanical system. The rest of the experiments were realised on MTs no. 2, 3 and 4 to examine model transferability by means of the difficulty of model modifications and the approximation quality achieved during both heating and cooling phases. MT no. 4 was also used as a target machine in [Mareš 2020, where only temperatures from the control system (motor) were available.

Tab. 3: List of experiments - movement in the $Y$ axis.

\begin{tabular}{cccc}
\hline $\begin{array}{c}\text { MT } \\
\text { no. }\end{array}$ & $\begin{array}{c}\text { Axis velocity } \\
\boldsymbol{V}_{\mathbf{Y}}\left[\mathrm{mm}_{\mathrm{min}}^{-1}\right]\end{array}$ & $\begin{array}{c}\text { Measured } \\
\text { phases }\end{array}$ & $\begin{array}{c}\text { Measured input } \\
\text { key temperatures }\end{array}$ \\
\hline 1 & 15000 & heating & $\mathrm{T}_{\text {nut, }}, \mathrm{T}_{\text {flange, }}, \mathrm{T}_{\text {motor }}$ \\
2 & 8000 & heating, cooling & $\mathrm{T}_{\text {nut }}, \mathrm{T}_{\text {flange }}, \mathrm{T}_{\text {motor }}$ \\
3 & 15000 & heating, cooling & $\mathrm{T}_{\text {nut }}, \mathrm{T}_{\text {flange, }}, \mathrm{T}_{\text {motor }}$ \\
4 & 15000 & heating, cooling & $\mathrm{T}_{\text {motor }}$ \\
\hline
\end{tabular}




\subsection{Model modifications}

The gain of the model outputs is the only used modification. This approach allows for a clear comparison of the four target machines from a single production line:

sim.ZTY inut $=\Delta T_{\text {iY nut }} \cdot \varepsilon_{Y \text { nut }} \cdot g_{\text {iY nut }}$,

sim. $Z T Y_{i \text { flange }}=\Delta T_{i Y \text { flange }} \cdot \varepsilon_{Y \text { flange }} \cdot g_{i Y \text { flange }}$,

sim.ZTY $Y_{\text {motor }}=\Delta T_{i Y \text { motor }} \cdot \varepsilon_{Y \text { motor }} \cdot g_{i Y \text { motor }}$,

where $i$ denoted the target machine number (2, 3 or 4$)$ and $g_{\mid \text {Y nut, }} g_{\mid \text {Y flange and }} g_{\mid \text {Y motor }}$ are the gain factors of model outputs. The gain factor values were selected based on the best fit. Table 4 summarises the gain factors.

Tab. 4: Gain factors ' $g$ ' of ZTY model modifications.

\begin{tabular}{ccccc}
\hline Machine tool & \multicolumn{3}{c}{$Y$ axis movement } \\
no. & $T_{\text {nut }}$ & & $T_{\text {flange }}$ & $T_{\text {motor }}$ \\
\hline 1 & 1 & 1 & 1 \\
2 & 1.7 & 2.7 & 1.5 \\
3 & 2.5 & 2.4 & 1.6 \\
4 & - & - & 1.1 \\
\hline
\end{tabular}

The gain factors for the models using flange temperature inputs are higher than the factors used in the models with motor and nut temperatures. This preliminary observation indicates $T_{\text {nut }}$ and $T_{\text {motor }}$ as better input values for compensation models. The gain factors for MT no. 3 models are also higher than the factors for MT no. 2 and MT no. 4. This result could indicate lower model effectivity when applied to a 'worn' machine. Detailed model application results are the subject of the next section.
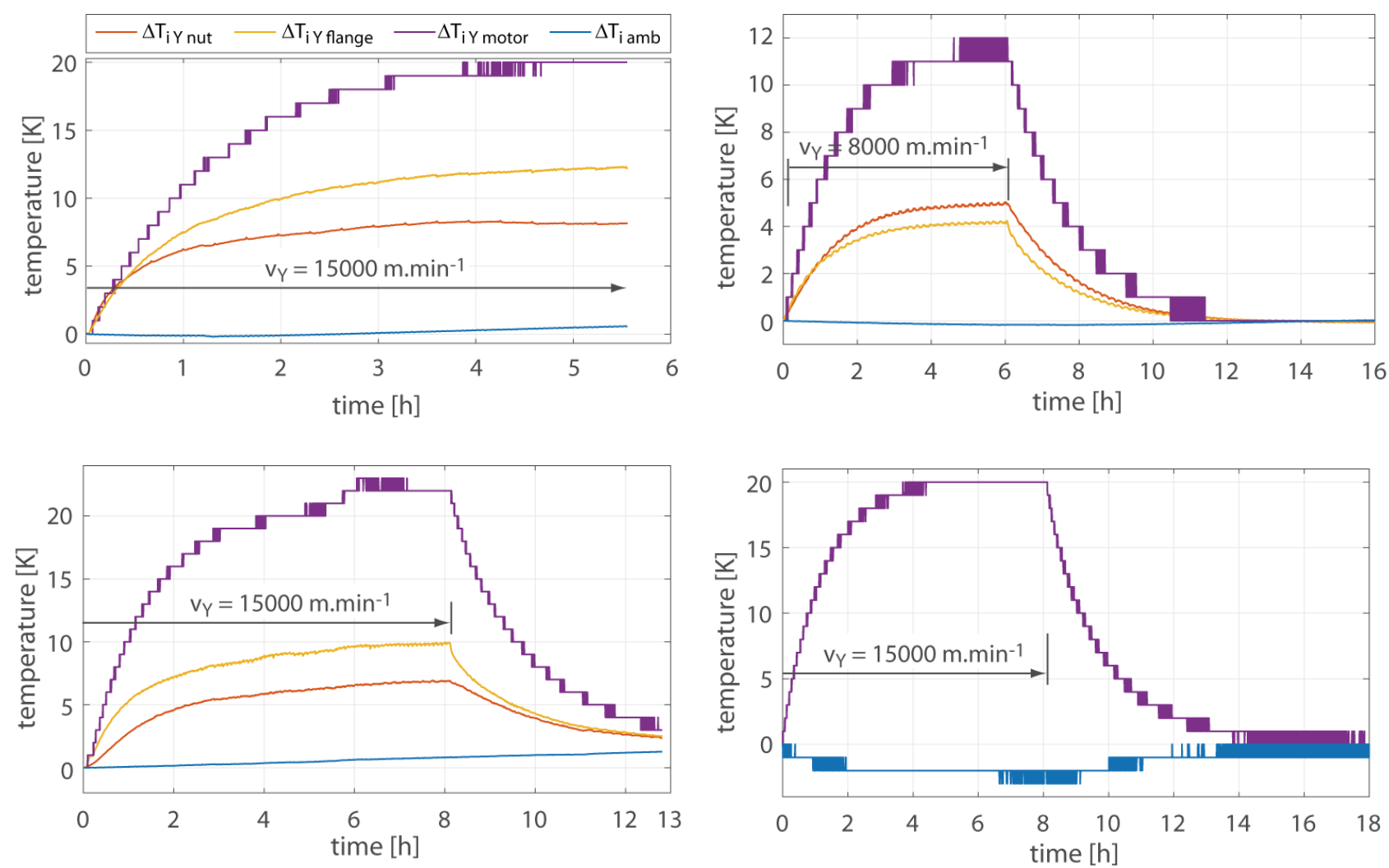

Fig. 8: Measured temperature inputs during verification tests of $Y$-axis movement impact on MT no.1 (upper left), MT no.2 (upper right), MT no.3 (bottom left), MT no.4 (bottom right). 

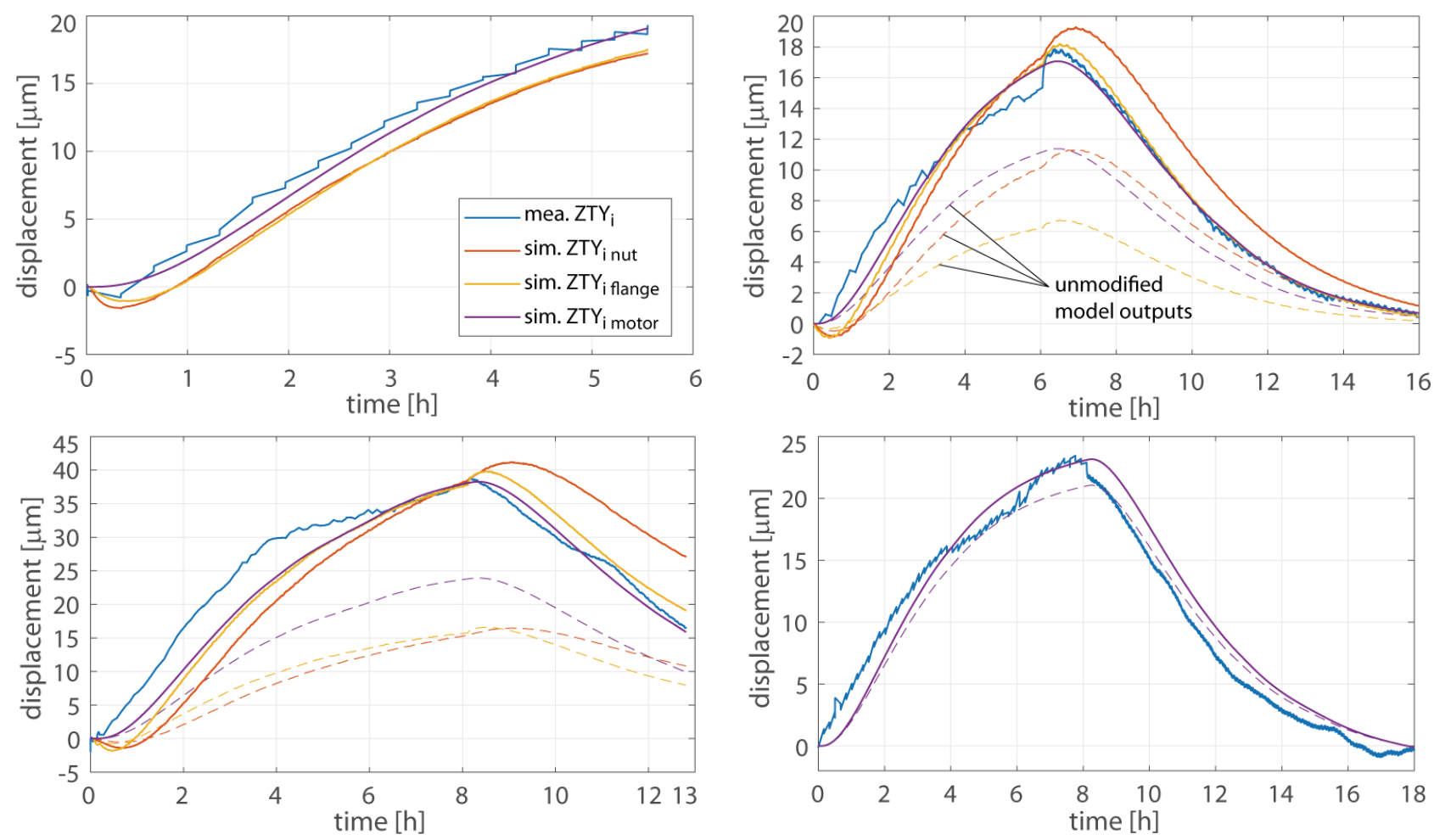

Fig. 9: Measured and simulated deformations during verification tests of $Y$-axis movement impact on MT no.1 (upper left), MT no.2 (upper right), MT no.3 (bottom left), MT no.4 (bottom right).

The linear dependency between the inputs and outputs of the thermo-mechanical system may be observed in Fig. 9 (upper left), where all of the unmodified models show very good approximation quality during the entire behaviour of the verification test performed on MT no. 1. The model with motor temperature input shows the best result in transient behaviour description. Similar outcomes are observable from other tests performed on MTs no. 2, 3 and 4, where the model with motor temperature input also approximates cooling phases very well. Temperatures from other key points (nut and flange) are not beneficial for modelling ZTY thermal behaviour. The time constants of transient behaviours differ slightly across all of the tests (we observed that the models' approximation abilities were worse at the beginning of the experiments). This fact, and the necessity of gain factors, point out differences caused by assembly and production inaccuracies in MT components in the target machine production line. The only transfer of the unmodified model that showed satisfactory results was between MT no. 1 and MT no. 4. In contrast, the worst approximation quality and model transferability was observed during application on MT no. 3, a machine used in a real production process. The last result indicates time-dependent changes in MT components caused by their use.

\section{DISCUSSION}

Thermal errors caused by movement in the $Z$ linear axis ZTZ and compensation effectivity are discussed in this section. The experiment set-up for $Z$-axis movement impact inspection (with all positions, trajectories and speeds) is depicted in Fig. 10.

Approximation models of ZTZ deformations were constructed for MT no. 1 following the same procedure as described in section 3 (the axis speed was set to a constant $v_{z}=10000 \mathrm{~mm} \cdot \mathrm{min}^{-1}$ per 8 hours - heating phase only, and the model structures are similar). Two key temperatures were selected as model inputs (flange and motor). The identified models achieved 92\% (flange) and 93\% (motor) of fit approximation quality during the calibration process.

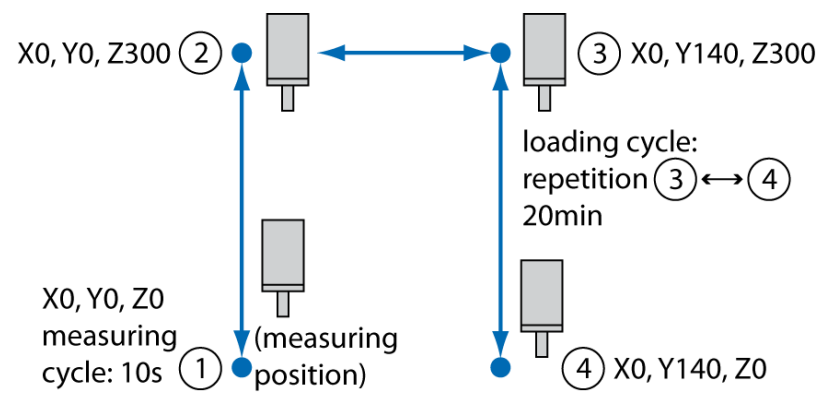

Fig. 10: Experiment set-up for calibration of $Z$-axis movement impact on MT thermal behaviour.

Two target machines (no. 2 and no. 3) were chosen for verification experiments with $Z$-axis activity. Key temperatures of flange and motor (best key temperature points resulting from ZTY analyses) were measured on both machines. Heating and cooling phases were recorded during the experiments. Machine no. 2 was brand new in the testing period; machine no. 3 was used in a production process. A list of two verification experiments with $Z$-axis movement is presented in Table 6 .

Tab. 6: List of experiments - Z-axis movement.

\begin{tabular}{cccc}
\hline $\begin{array}{c}\text { MT } \\
\text { no. }\end{array}$ & $\begin{array}{c}\text { Axis velocity } \\
\text { V }\left[\mathrm{mm}_{\mathrm{min}} \mathrm{m}^{-1}\right]\end{array}$ & $\begin{array}{c}\text { Measured } \\
\text { phases }\end{array}$ & $\begin{array}{c}\text { Measured input } \\
\text { key temperatures }\end{array}$ \\
\hline 2 & 8000 & heating, cooling & $\mathrm{T}_{\text {flange }}, \mathrm{T}_{\text {motor }}$ \\
3 & 15000 & heating, cooling & $\mathrm{T}_{\text {flange, }}, \mathrm{T}_{\text {motor }}$ \\
\hline
\end{tabular}

Gain factors modifying the compensation models for application to a machine other than the calibrated were necessary for ZTZ deformations similarly to ZTY. The gain factors for MT no. 2 and no. 3 are presented in Table 7. 
Tab. 7: Gain factors ' $g$ ' of ZTZ model modifications.

\begin{tabular}{ccc}
\hline \multirow{2}{*}{ Machine tool no. } & \multicolumn{2}{c}{ Z axis movement } \\
& $T_{\text {flange }}$ & $T_{\text {motor }}$ \\
\hline 2 & 1.1 & 0.9 \\
3 & 2.1 & 1.6 \\
\hline
\end{tabular}

The gain factors for MT no. 3 models are higher (especially in models using flange temperature inputs) than the factors for MT no. 2. A similar result was observed in the previous $Y$-axis movement section.

The results of the two tests with models transferred among target machines no. 1, 2 and 3 from global (fit) and loca $(p 2 p)$ approximation quality perspectives are summarised in Table 8.

Tab. 8: Results of ZTZ models and transferability among target machines 1, 2 and 3.

\begin{tabular}{|c|c|c|c|}
\hline \multirow{3}{*}{$\begin{array}{l}\text { MT } \\
\text { no. }\end{array}$} & \multirow{3}{*}{$\begin{array}{c}\text { Axis } \\
\text { velocity } \\
\text { Vz } \\
{\left[\mathrm{mm} \cdot \mathrm{min}^{-1}\right]}\end{array}$} & \multicolumn{2}{|c|}{ Key temperature in $Z$ axis } \\
\hline & & $T_{\text {flange }}$ & $T_{\text {motor }}$ \\
\hline & & \multicolumn{2}{|c|}{ Global approx. quality fit [\%] } \\
\hline 2 & 8000 & 85 & 84 \\
\hline \multirow[t]{2}{*}{3} & 15000 & 73 & 64 \\
\hline & & \multicolumn{2}{|c|}{$\begin{array}{l}\text { Local approx. quality } p 2 p[\mu \mathrm{m}] \\
\text { (original/compensated) }\end{array}$} \\
\hline 2 & 8000 & $17 / 2.9$ & $17 / 3.6$ \\
\hline 3 & 15000 & $59.8 / 14.6$ & $59.8 / 18.8$ \\
\hline
\end{tabular}

The measured key point temperature inputs (flange, and motor) into the thermal error models during the Z-axis movement verification experiments performed on MTs no. 2 and 3 are depicted in Fig. 11.
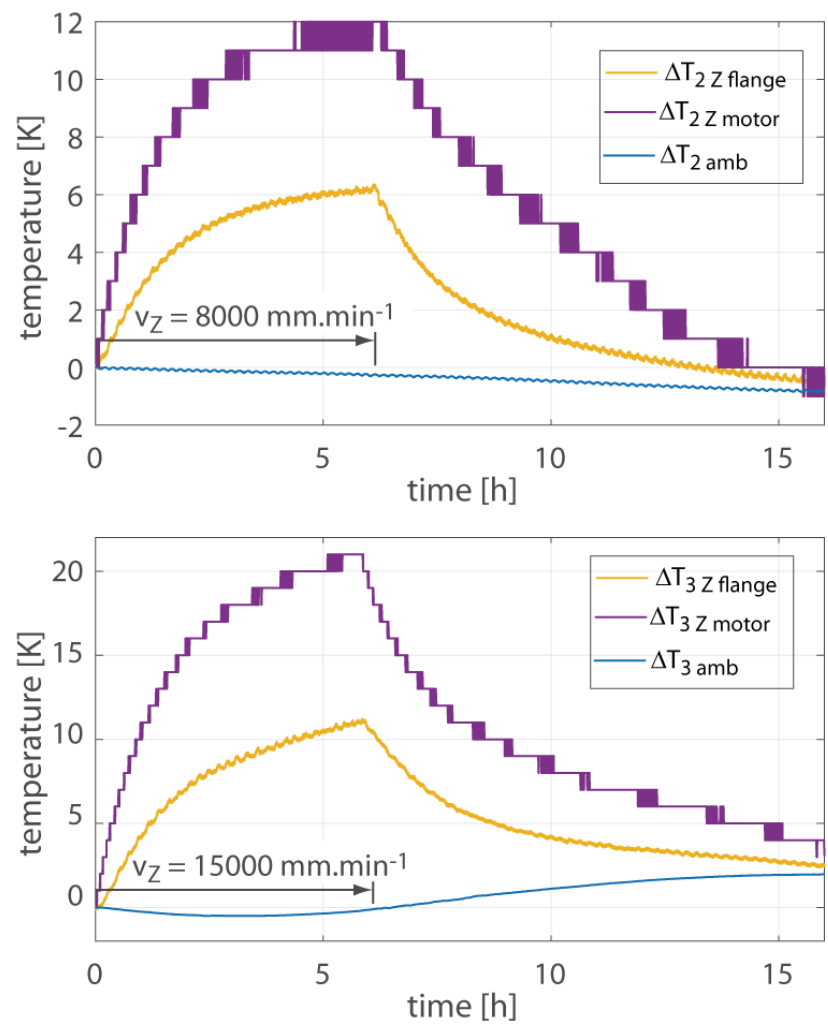

Fig. 11: Measured temperature inputs during verification tests of Z-axis movement impact on MT no.2 (upper) and MT no.3 (bottom).
The detailed results of modified model applications are presented in the following graphs (Fig. 12 (upper) for MT no. 2 and Fig. 12 (bottom) for MT no. 3).
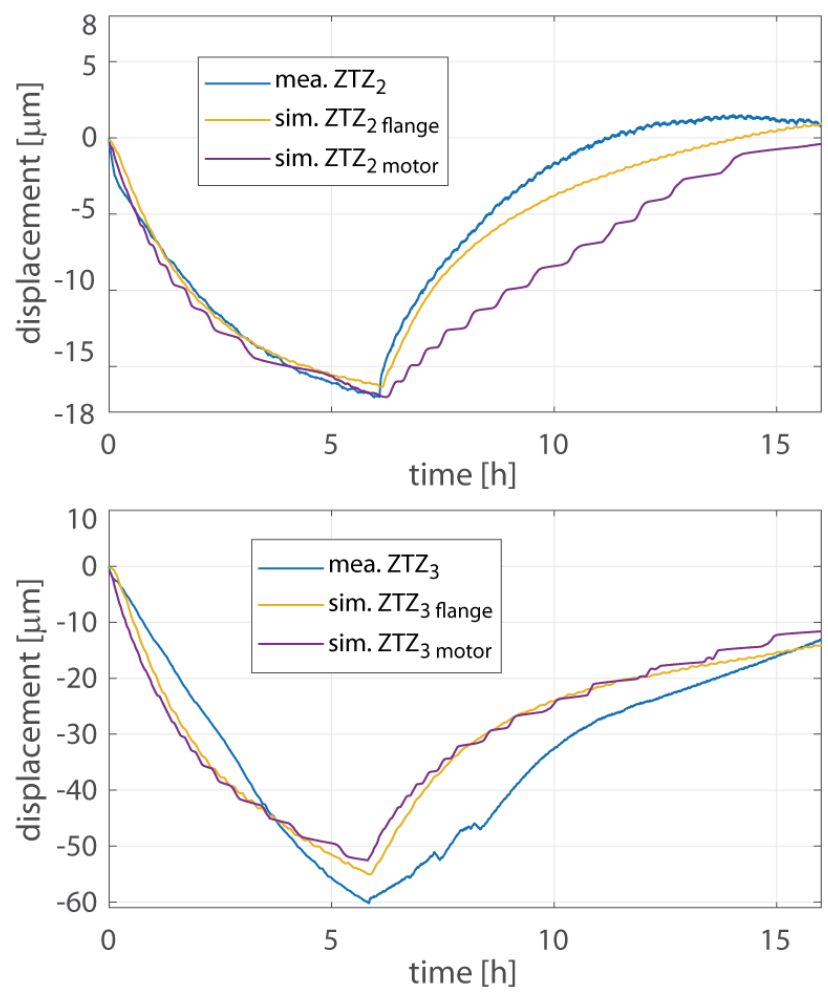

Fig. 12: Measured and simulated deformations during verification tests of Z-axis movement impact on MT no.2 (upper) and MT no.3 (bottom).

Contrary to the ZTY results, the flange key temperature point shows better approximation quality in the cooling phase of ZTZ, as can be seen in Fig. 12 (upper). Independent cooling from other MT parts of the motor might cause the difference. The motor flange temperature input has better results during application of the modified model to MT no. 3, as evident from the global and local approximation quality point of view (Table 8 ). The worse model approximation quality of machine no. 3 confirms the assumption of nonlinear time-dependent changes in MT components caused by their use. A new TF should be identified if there is a need to further increase MT no. 3 model accuracy.

\section{CONCLUSIONS}

A critical point of any compensation model development is selection of suitable inputs into the thermo-mechanical system and its industrial applicability. The main objective of the investigation presented in this article is analysis of suitable key temperature points as thermal error model inputs and complexity of model portability between different machines of the same production line.

The four tested machines were gantry type 5-axis milling centres differing in the state of wear (three brand new machines and one machine used in a production process), adjustment of components (preloading of ball-screws, lubrication, etc.) and year of manufacture. The machines were loaded by $Y$ - and $Z$-axis movements and thermal errors ZTY and ZTZ were inspected. Three key temperature points were compared as suitable inputs to thermal error compensation models: motor temperature (embedded in the MT control system with an indirect relationship to thermal errors), ball-screw nut (placed on moving parts of

MM Science Journal | 2021 | NOVEMBER - Special Issue on HSM2021 
the machine with assumed good correlation with thermal errors caused by moving linear axes) and motor flange temperature (stationary part of the MT firmly connected to its structure and close to heat sources caused by motor and ball-screw activities).

A set of compensation models based on transfer functions was developed on one target machine and verified on the rest with the following results:

- The linear dependency between the inputs and outputs of the thermo-mechanical system was demonstrated by changes in axis speed with no need to make model modifications in one target machine.

- Gain factors were necessary to model adjustments during application to different machines. This result points to the influence of assembly and production inaccuracies of machine components (with the accuracy of the location of the external temperature sensors taken into account). It is impossible to evaluate the impact of each influence despite the known differences between the target machines defined in Table 1. More extensive research should be carried out even on level of MT components.

- The time constants of the inspected machine thermal behaviours differed slightly as well. The average efficiency of the transferred models to the brand new machines was $80 \%$ and $65 \%$ for transfer to the machine used in a production process. The results were similar for both ZTY and ZTZ thermal errors.

- Model inputs from motor and motor flange key temperature points resulted in better approximation quality than ball-screw nut. Approximation quality differences between the inputs were observed in particular during the cooling phases.

From the perspective of modern MT trends, compensation models are a necessary part of smart solutions for intelligent MT and manufacturing. Such solutions rest on an assumption of long-term stability and should have attributes suitable for machine learning principles. Compensation models have to be verified in real industrial conditions to guarantee their practical applicability. An evaluation of model functionality within spatial positioning error and model integration into hybrid compensation principles (a beneficial combination with in-process measurements) will be the focus of recent and follow-up research.

\section{ACKNOWLEDGMENTS}

This work was supported by the Grant Agency of CTU in Prague, grant no. SGS19/165/OHK2/3T/12.

\section{REFERENCES}

[Hernandez-Becerro 2018] Hernández-Becerro, P., et al., Model order reduction of thermal models of machine tools with varying boundary conditions. In: CIRP-sponsored 1st Conference on Thermal Issues in Machine Tools, Dresden, Germany, 21-23 March 2018.

[Blaser 2019] Blaser, P., et al., Long-term thermal compensation of 5-axis machine tools due to thermal adaptive learning control, MM Science Journal, 2019, pp 3164-3171. Available online: https://www.mmscience.eu/en [Brecher 2004] Brecher, C, et al., Compensation of thermoelastic machine tool deformation based on control internal data. CIRP Annals - Manufacturing Technology, 2004, Vol. 53, No. 1, pp 299-304.
[Hellmich 2018] Hellmich, A.,et al., 2018, Methods for analyzing and optimizing the fluidic tempering of machine tool beds of high performance concrete. In: CIRPsponsored 1st Conference on Thermal Issues in Machine Tools, Dresden, Germany, 21-23 March 2018.

[Horejs 2020] Horejs, O., et al., Real-time compensation of a 5-axis CNC milling centre thermal errors considering different spindle units. In Conference Proceedings - 20th International Conference of the European Society for Precision Engineering and Nanotechnology, Geneve, Switzerland, 2020, pp. 559-562.

[ISO 230-3 2007] ISO 230-3 Test code for machine tools Part 3: Determination of thermal effects. International Organization for Standardization ISO, Geneva, Switzerland, 2007, p. 44.

[Li 2008] Li, J.W., et al., Thermal-error modeling for complex physical systems: the-state-of-arts review, Int J Adv Manuf Technol, 2009, Vol. 42, pp 168-179.

[Liu 2019] Liu, K., et al., Intelligentization of machine tools: comprehensive thermal error compensation of machineworkpiece system. The International Journal of Advanced Manufacturing Technology, 2019, 102(9-12), pp 38653877.

[Ljung 2020] Ljung, L. System Identification Toolbox ${ }^{\mathrm{TM}}$ User's Guide. The MathWorks, 2015. https://ch.mathworks.com/help/pdf doc/ident/ident ug.pdf

[Mares 2019] Mares, M., et al., Milling centre thermal compensation strategy based on transfer functions validated out of calibration range. MM Science Journal, Special Issue on HSM2019, November 2019, pp 31563163.

[Mares 2020] Mares, M., et al., Thermal error compensation of a 5-axis machine tool using indigenous temperature sensors and CNC integrated Python code validated with a machined test piece. Precision Engineering, November 2020, Vol.66, pp 21-30.

[Mayr 2012] Mayr, J., et al., Thermal issues in machine tools. CIRP Annals - Manufacturing Technology, 2012, Vol. 61, No. 2, pp 771-791.

[Mayr 2018] Mayr, J., et al., An Adaptive Self-Learning Compensation Approach for Thermal Errors on 5-Axis Machine Tools Handling an Arbitrary Set of Sample Rates, CIRP Annals - Manufacturing Technology, 2018, Vol. 67, pp 551-554.

[Mize 2000] Mize, C., et al., Neural network thermal error compensation of a machining center, Prec. Eng., 24/4, 2000, pp 338-346.

[Naumann 2018] Naumann, C., et al., Hybrid correction of thermal errors using temperature and deformation sensors. In: CIRP-sponsored 1st Conference on Thermal Issues in Machine Tools, Dresden, Germany, 21-23 March 2018.

[Srinivas 2017] Srinivas, N. G., et al., Optimization of high speed machine tool spindle to minimize thermal distortion. Procedia CIRP 58, 2017, pp 457-462.

[Zimmermann 2020] Zimmermann, N., et al., Extended discrete R-Test as on-machine measurement cycle to separate the thermal errors in Z-direction. Proceedings of the euspen's Special Interest Group: Thermal Issues, Aachen, Germany, 26-27 February 2020, pp 21-24. 